\title{
Magneto-ionic control of interfacial magnetism
}

\author{
Uwe Bauer ${ }^{1}$, Lide Yao ${ }^{2}$, Aik Jun Tan ${ }^{1}$, Parnika Agrawal', Satoru Emori ${ }^{1}$, Harry L. Tuller', \\ Sebastiaan van Dijken ${ }^{2}$ and Geoffrey S. D. Beach ${ }^{1 \star}$
}

\begin{abstract}
In metal/oxide heterostructures, rich chemical ${ }^{1,2}$, electronic ${ }^{3-5}$, magnetic $^{6-9}$ and mechanical ${ }^{10,11}$ properties can emerge from interfacial chemistry and structure. The possibility to dynamically control interface characteristics with an electric field paves the way towards voltage control of these properties in solid-state devices. Here, we show that electrical switching of the interfacial oxidation state allows for voltage control of magnetic properties to an extent never before achieved through conventional magneto-electric coupling mechanisms. We directly observe in situ voltage-driven $\mathrm{O}^{2-}$ migration in a $\mathrm{Co} / \mathrm{metal}$-oxide bilayer, which we use to toggle the interfacial magnetic anisotropy energy by $>0.75 \mathrm{erg} \mathrm{cm}^{-2}$ at just $2 \mathrm{~V}$. We exploit the thermally activated nature of ion migration to markedly increase the switching efficiency and to demonstrate reversible patterning of magnetic properties through local activation of ionic migration. These results suggest a path towards voltage-programmable materials based on solid-state switching of interface oxygen chemistry.
\end{abstract}

The physical and chemical properties of nanoscale materials derive largely from structure and composition at interfaces. The possibility to electrically modify these interfacial characteristics would provide a powerful means to control material properties. Of particular recent scientific and technological interest are metal/metaloxide bilayers $^{1-15}$, in which properties as varied as catalytic activity ${ }^{1,2}$, charge and spin transport ${ }^{3-6}$, ionic exchange $e^{14,15}$, mechanical behaviour $^{10,11}$, thermal conductivity ${ }^{12,13}$ and magnetism ${ }^{6-9}$ all depend sensitively on oxygen stoichiometry and defect structure at the metal/metal-oxide interface. Ionic transport in metal oxides can be driven by an electric field, and $\mathrm{O}^{2-}$ migration is already exploited as a mechanism for resistive switching in anionic metal/oxide/metal memristors ${ }^{3,4}$. However, the broader application of voltage-driven oxygen transport to control interfacial phenomena in metal/metaloxide structures is only beginning to be explored.

For example, ferromagnetic metal/metal-oxide bilayers such as $\mathrm{Co} / \mathrm{AlO}_{x}$ and $\mathrm{CoFe} / \mathrm{MgO}$ exhibit strong perpendicular magnetic anisotropy (PMA) derived from interfacial Co-O hybridization ${ }^{8,9}$. The ability to toggle interfacial PMA with a gate voltage would markedly reduce switching energies in spintronic devices, and could enable new device architectures exploiting local gating of magnetic properties ${ }^{16-26}$. Most work on voltage control of magnetic anisotropy in metal/metal-oxide bilayers has focused on charge accumulation or band shifting in the metal layer ${ }^{16-19}$. However, experimental reports of irreversibility, and anisotropy changes much larger than theoretically predicted ${ }^{20,21}$, suggest that ionic effects may be important and in some cases even dominant ${ }^{22-26}$. Nonetheless, electric-field-driven oxygen migration in metal/metaloxide bilayers is difficult to observe directly, and the impact on magnetic properties has not yet been established.
Here we report direct in situ observation of voltage-driven $\mathrm{O}^{2-}$ migration in a metal/metal-oxide bilayer, and show that solid-state electro-chemical switching of the interfacial oxidation state can be used to completely remove and restore PMA in a thin Co layer. Using cross-sectional transmission electron microscopy (TEM) and high-resolution electron energy-loss spectroscopy (EELS), we track in situ voltage-driven migration of the oxidation front in a $\mathrm{Co} / \mathrm{GdO}_{x}$ bilayer. By varying temperature and interface structure, we relate motion of the oxidation front to voltageinduced anisotropy changes, and show that by minimizing the $\mathrm{O}^{2-}$ diffusion barrier, the timescale for magneto-ionic switching can be reduced by $\sim 6$ orders of magnitude. We toggle interfacial PMA by an unprecedented $>0.75 \mathrm{erg} \mathrm{cm}^{-2}$ at just $2 \mathrm{~V}$, and achieve a magneto-electric efficiency $>5,000 \mathrm{fJ} \mathrm{V}^{-1} \mathrm{~m}^{-1}$. Finally, we introduce a general method to reversibly imprint material properties through local activation of ionic migration, which we use to locally pattern magnetic anisotropy and create domain wall (DW) conduits in continuous magnetic films. These results establish magneto-ionic coupling as a powerful mechanism for voltage control of magnetism.

Experiments focus on $\mathrm{Ta}(4 \mathrm{~nm}) / \mathrm{Pt}(3 \mathrm{~nm}) / \mathrm{Co}(0.9 \mathrm{~nm}) / \mathrm{GdO}_{x}$ ( $3 \mathrm{~nm}$ ) films sputter-deposited on thermally oxidized Si (Methods). The films exhibit strong PMA with an in-plane saturation field $H_{\mathrm{k}} \sim 10 \mathrm{kOe}$ (Supplementary Fig. 3). Square $200 \mu \mathrm{m} \times 200 \mu \mathrm{m}$ $\mathrm{GdO}_{x}(30 \mathrm{~nm}) / \mathrm{Ta}(2 \mathrm{~nm}) / \mathrm{Au}(12 \mathrm{~nm})$ gate electrodes were patterned on top of the film for voltage application, with the bottom metal stack used as a counter electrode. Figure 1a shows a high-resolution cross-sectional TEM micrograph of the layer structure, with the thin Co layer embedded between polycrystalline Pt and $\mathrm{GdO}_{x}$ layers.

Spatially resolved EELS experiments were carried out in scanning TEM (STEM) mode on the same cross-section to measure the chemical profile and to detect changes induced by a gate voltage $V_{\mathrm{g}}$. Voltage was applied by contacting the top electrode in situ with a $\mathrm{Pt} / \mathrm{Ir}$ tip as shown in Fig. 1a, inset (see Supplementary Information for details). Figure $1 \mathrm{~b}$ shows representative STEMEELS spectra with $\mathrm{O} \mathrm{K}$-edges and $\mathrm{Co}$ white lines from a location in the centre of the Co layer. The evolution of the O K-edge and Co $\mathrm{L}_{3}$-edge count rates along a line profile perpendicular to the layers is shown in Fig. 1c. The Co layer is clearly distinguished, with no $\mathrm{O}$ detected within the Co layer and a sharp rise in the $\mathrm{O}$ signal at the $\mathrm{Co} / \mathrm{GdO}_{x}$ interface. STEM-EELS scans were then performed after applying a negative bias, which drives $\mathrm{O}^{2-}$ towards the Co layer. The appearance of an $\mathrm{O} \mathrm{K}$-edge signal at the centre of the Co layer can be inferred from the spectra in Fig. 1b, taken after applying $-3 \mathrm{~V}$ for $\sim 100 \mathrm{~s}$. After subsequently applying $-5 \mathrm{~V}$ for $\sim 100$ s, the STEM-EELS scan in Fig. 1d shows that O penetrates all the way to the bottom $\mathrm{Co} / \mathrm{Pt}$ interface. Figure $1 \mathrm{e}, \mathrm{f}$ demonstrates that Co oxidation is reversible under positive bias. Figure 1e shows a STEM-EELS scan after first applying a negative bias of $-3 \mathrm{~V}$ until 


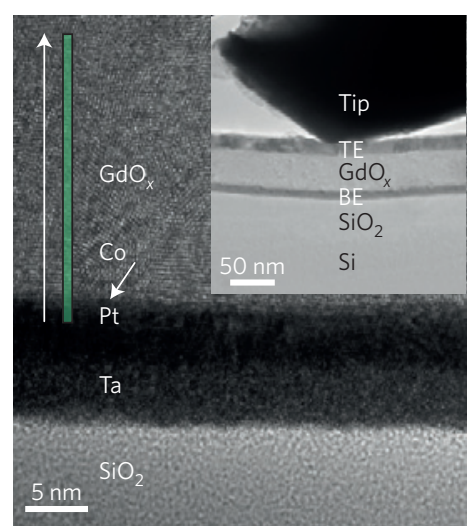

d

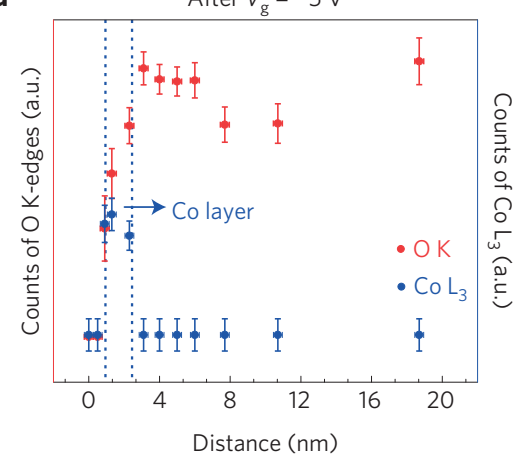

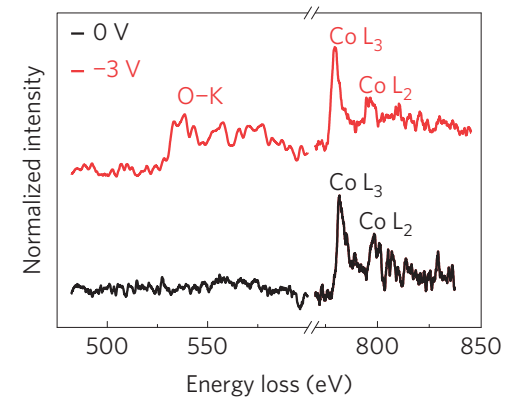

e

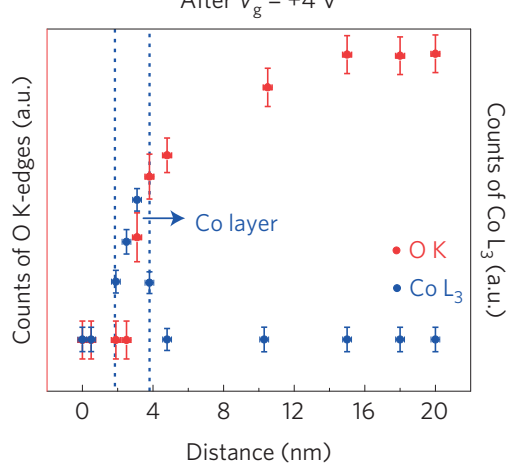

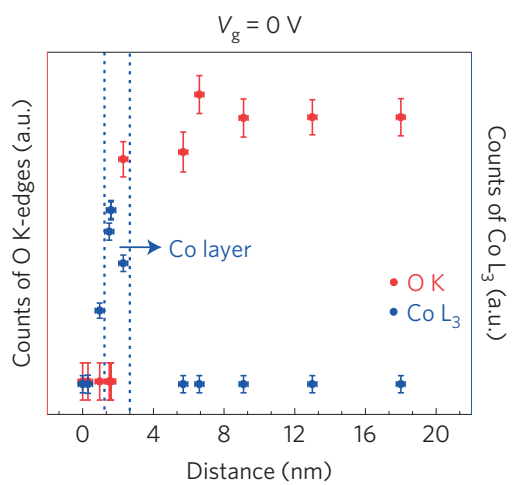

f

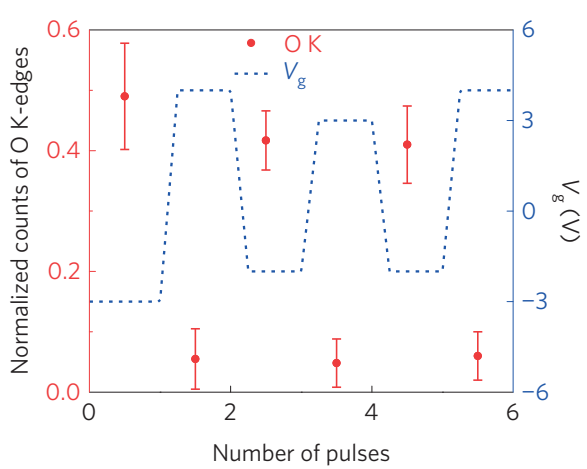

Figure 1 | Cross-sectional TEM and EELS analysis. a, High-resolution TEM image of the $\mathrm{SiO}_{2} / \mathrm{Ta}_{\mathrm{P}} \mathrm{Pt} / \mathrm{Co}_{\mathrm{GG}} \mathrm{G}$ layer structure. STEM-EELS spectra were measured along the green line, starting from the Pt/Co interface as indicated by the white arrow. Inset: contact between the Pt/Ir probe of the in situ TEM

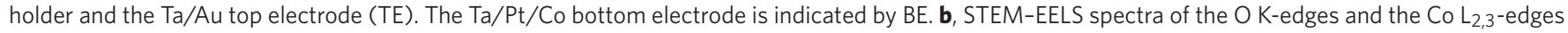

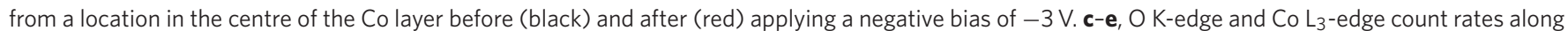
a similar line profile as indicated in $\mathbf{a}$. The data are obtained before the application of a bias voltage (c), after applying - $5 \mathrm{~V}$ (d), and after first applying

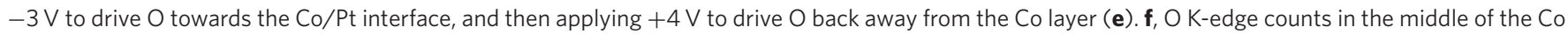
layer normalized to the $\mathrm{O} \mathrm{K}$-edge signal in the $\mathrm{GdO}_{x}$ layer following three cycles of alternating positive and negative bias voltage. The error bars in this figure represent the intrinsic EELS measurement noise and uncertainty during background subtraction.

an $\mathrm{O}$ signal was detected near the $\mathrm{Co} / \mathrm{Pt}$ interface, and then applying $V_{\mathrm{g}}=+4 \mathrm{~V}$ for $\sim 100 \mathrm{~s}$, which drives oxygen away from the Co layer (see Supplementary Information for details). Finally, Fig. 1f shows that the $\mathrm{O}^{2-}$ content in the Co layer can be toggled repeatedly by cycling $V_{\mathrm{g}}$ between alternating bias polarities.

We examined the impact of voltage-induced $\mathrm{O}^{2-}$ migration on magnetic properties using a scanning magneto-optical Kerr effect (MOKE) polarimeter with a $\sim 3 \mu \mathrm{m}$ laser spot to locally probe hysteresis characteristics. Figure 2a maps the coercivity $H_{c}$ in the vicinity of a gate electrode, shown schematically in Fig. $2 b$. Before measurement, a domain nucleation site was created nearby using a mechanical microprobe ${ }^{22,23,27}$ so that $H_{\mathrm{c}}$ represents the DW propagation field ${ }^{22,23,27}$, which is highly sensitive to the magnetic anisotropy energy (MAE) landscape.

In the virgin state (Fig. $2 \mathrm{a}$ ), $H_{\mathrm{c}}$ is uniform across the measured area, reflecting a DW propagation field of $\sim 200$ Oe due to finescale disorder. After applying $V_{\mathrm{g}}=-4 \mathrm{~V}$ for $240 \mathrm{~s}$ and then setting $V_{\mathrm{g}}=0 \mathrm{~V}, H_{\mathrm{c}}$ exhibits an abrupt step at the electrode edge (Fig. 2c) and increases to $\sim 340$ Oe beneath the electrode. Similar behaviour has been observed previously ${ }^{23}$ and attributed to $\mathrm{O}^{2-}$ migration near the electrode perimeter, where ionic transport is typically most efficient ${ }^{28}$. This would locally reduce the MAE by over-oxidizing the Co interface ${ }^{8,9}$, creating potential wells at the electrode edge (Fig. 2d) that trap propagating DWs, increasing $H_{\mathrm{c}}$. We verified that the DW propagation field at the interior of the electrode remained unchanged after $V_{\mathrm{g}}$ application by mechanically creating a nucleation site inside the electrode. The observed magnetic behaviour is thus consistent with the schematic MAE landscape in Fig. 2d.

The lack of irreversible MAE changes at the electrode interior suggests that the timescale for bulk $\mathrm{O}^{2-}$ diffusion is much longer than at the electrode perimeter, where the open oxide edge (Fig. 2b) provides a high-diffusivity path ${ }^{28}$. The high ionic mobility observed in Fig. 1 is likewise probably aided by the high surface to volume ratio of the polished TEM specimen, because the activation energy for surface diffusion is typically lower than for bulk ${ }^{28}$. Voltage-induced $\mathrm{O}^{2-}$ migration, however, need not be limited to the oxide edge. Owing to the thermally activated nature of ion migration, voltage application at elevated temperature should result in exponentially higher $\mathrm{O}^{2-}$ drift velocities ${ }^{28}$, and activation of bulk $\mathrm{O}^{2-}$ migration on an observable timescale.

Figure 2e shows a $H_{\mathrm{c}}$ map after applying $V_{\mathrm{g}}=-4 \mathrm{~V}$ for $155 \mathrm{~s}$ at $T=100^{\circ} \mathrm{C}$, and then cooling the sample back to room temperature at $V_{\mathrm{g}}=0 \mathrm{~V}$. In this case, $H_{\mathrm{c}}$ outside the electrode is unchanged, showing that the elevated temperature alone does not irreversibly change the magnetic properties (Supplementary Fig. 4). However, beneath the electrode $H_{c}$ drops to $\sim 50 \mathrm{Oe}$, indicating that DWs nucleate and propagate there at a much lower field, and are impeded by an anisotropy step at the electrode edge. This implies a significant MAE reduction across the electrode area, shown schematically in Fig. 2f, consistent with overoxidation of the Co interface ${ }^{8,9}$. 


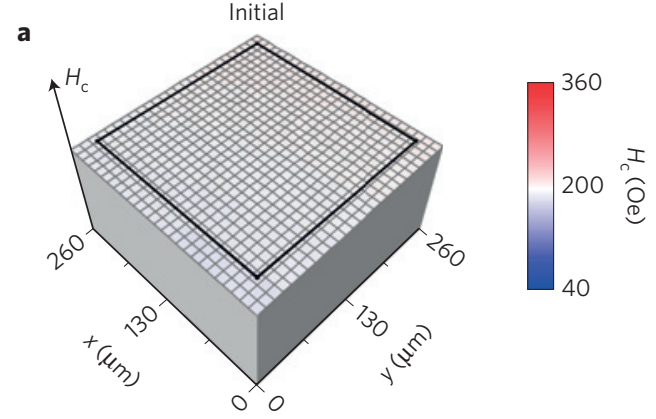

c

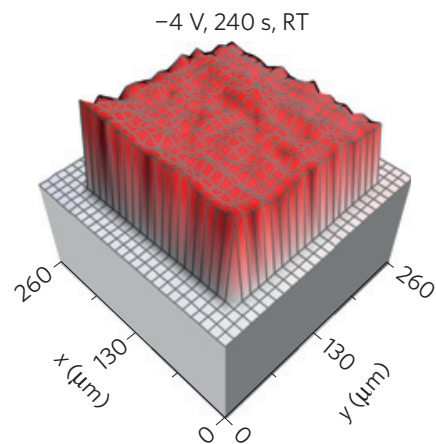

e

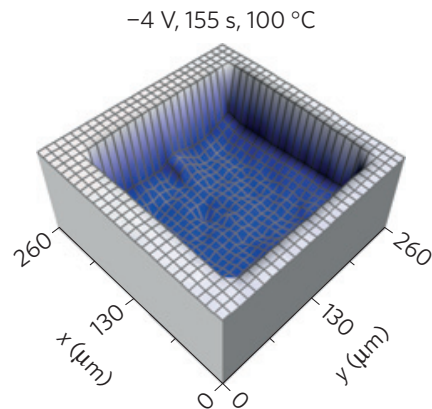

b

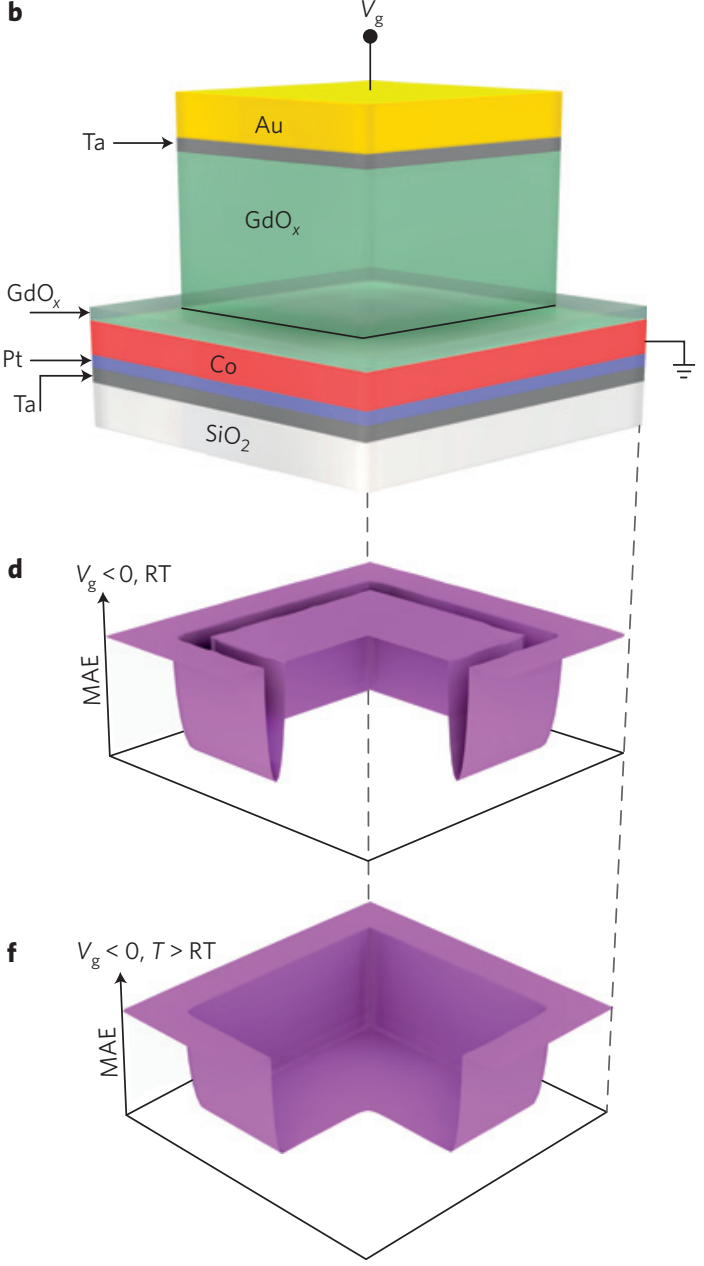

g

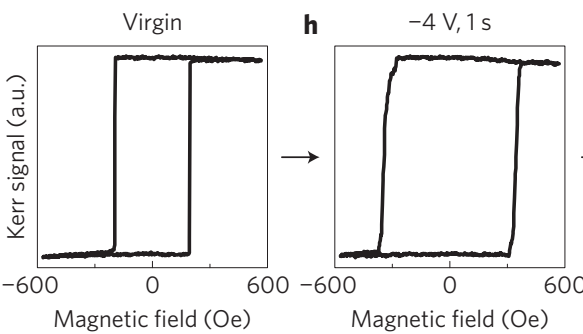

i

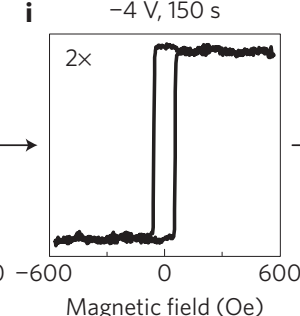

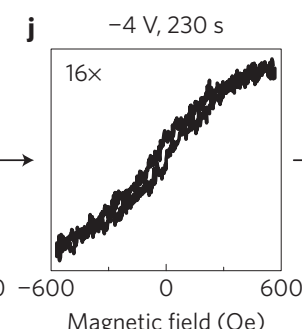

k

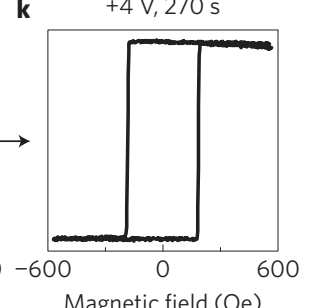

Figure 2 | Device schematics and voltage control of magnetic anisotropy. a, Topographic map of the coercivity $\left(H_{c}\right)$ in the virgin state, in the vicinity of a gate electrode. $\mathbf{b}$, Schematic view of gate-electrode structure. $\mathbf{c}, \mathbf{d}, H_{\mathrm{c}}$ and the implied MAE landscape, respectively, after applying a gate voltage $V_{\mathrm{g}}=-4 \mathrm{~V}$ for $240 \mathrm{~s}$ at room temperature (RT). e,f, The same as in $\mathbf{c}, \mathbf{d}$ after applying $V_{\mathrm{g}}=-4 \mathrm{~V}$ for $155 \mathrm{~s}$ at $100^{\circ} \mathrm{C}$. $\mathbf{g}-\mathbf{k}$, Polar MOKE hysteresis loops measured at room temperature at the centre of the gate electrode showing the device in its virgin state $(\mathbf{g})$, after applying $V_{\mathrm{g}}=-4 \mathrm{~V}$ at $100^{\circ} \mathrm{C}$ for $1 \mathrm{~s}(\mathbf{h}), 150 \mathrm{~s}(\mathbf{i})$ and $230 \mathrm{~s}(\mathbf{j})$, and after applying $V_{g}=+4 \mathrm{~V}$ at $100^{\circ} \mathrm{C}$ for $270 \mathrm{~s}(\mathbf{k})$. Note that the Kerr signal intensity in $\mathbf{i}$ is reduced by a factor of 2 and in $\mathbf{j}$ by a factor of 16 , as indicated by inset number.

As seen in Fig. 2g-k, the voltage-induced MAE change at $T=100^{\circ} \mathrm{C}$ is progressive. Here, we sequentially applied $V_{\mathrm{g}}=-4 \mathrm{~V}$ for a fixed dwell time at $T=100^{\circ} \mathrm{C}$, and then cooled the sample to room temperature with $V_{\mathrm{g}}=0 \mathrm{~V}$ to measure a hysteresis loop beneath the electrode. We observe an initial increase in $H_{\mathrm{c}}$ due to DW trap formation at the electrode perimeter, which occurs within $1 \mathrm{~s}$ of $V_{\mathrm{g}}$ application in contrast to the several minutes required at room temperature (Fig. 2c). With increasing voltage dwell time $H_{c}$ then drops precipitously, indicating a rapid reduction of PMA across the electrode. The saturation MOKE signal also declines, by nearly a factor of 2 after $\sim 150 \mathrm{~s}$ (Fig. 2i), suggesting increasing Co oxidation. After several minutes (Fig. 2j), PMA is lost entirely. Remarkably, PMA can be completely restored by reversing the bias polarity, as seen in Fig. 2k after applying $V_{\mathrm{g}}=+4 \mathrm{~V}$ for $270 \mathrm{~s}$ at $100^{\circ} \mathrm{C}$.

To correlate magnetic anisotropy with the location of the oxidation front, we used a Gd spacer to control the distance between $\mathrm{Co}$ and the $\mathrm{Gd} / \mathrm{GdO}_{x}$ interface. Here, $\mathrm{GdO}_{x}$ was grown as a continuous 30-nm-thick layer before depositing Ta/Au electrodes to prevent DW trap formation at the edges. Figure $3 \mathrm{a}-\mathrm{d}$ shows the 

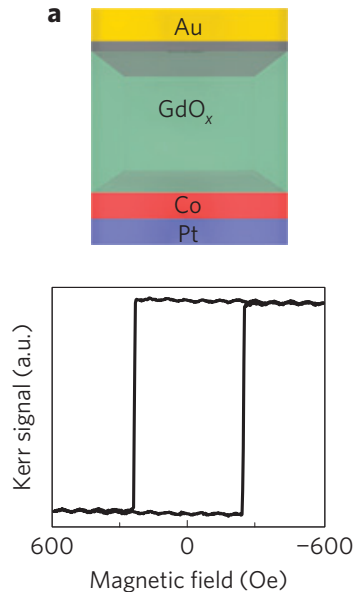
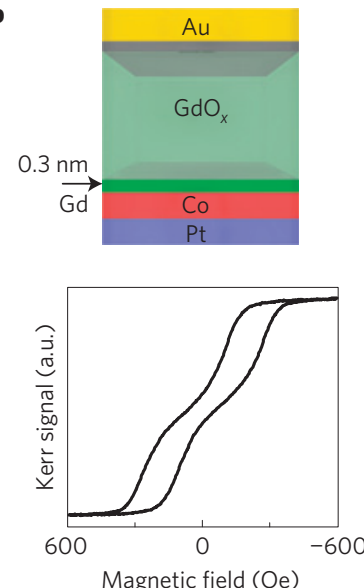
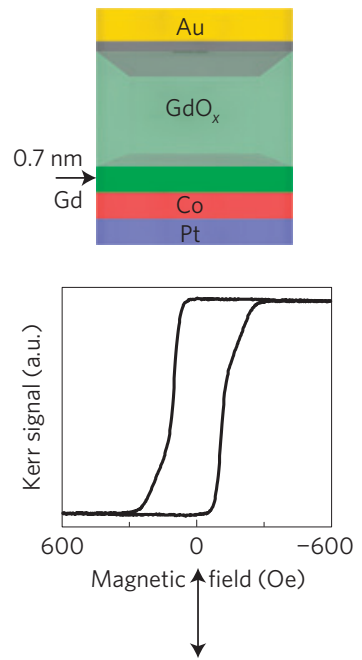
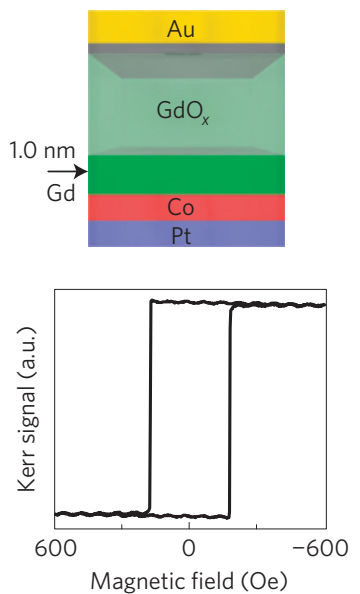

e

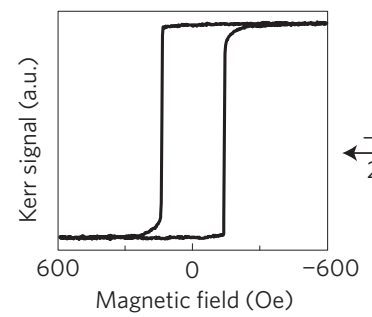

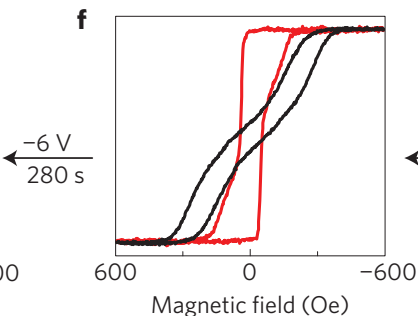

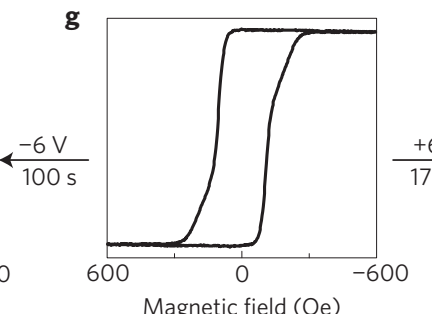

h

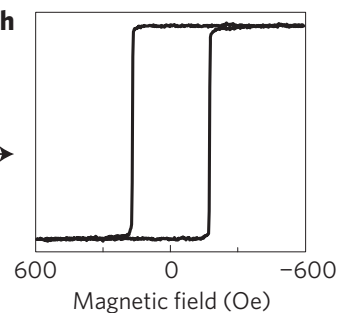

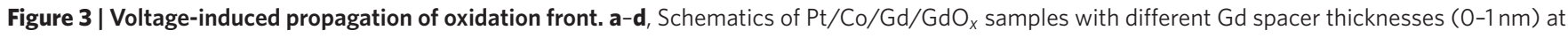
the $\mathrm{Co} / \mathrm{GdO}_{x}$ interface and polar MOKE hysteresis loops corresponding to the as-deposited samples. e-h, Evolution of polar MOKE hysteresis loops after application of positive $(\mathbf{e}, \mathbf{f})$ and negative $(\mathbf{h})$ gate voltage $V_{g}$ at $100^{\circ} \mathrm{C}$ to the sample with a 0.7 -nm-thick $\mathrm{Gd}$ spacer layer (c,g). All hysteresis loops were measured at room temperature and zero bias. The red curve in $\mathbf{f}$ shows a hysteresis loop after first setting the device in a state with minimum remanence ratio using a negative gate voltage, and then applying a $10 \mathrm{~ms}$ voltage pulse $V_{\mathrm{g}}=+12 \mathrm{~V}$ at $120^{\circ} \mathrm{C}$, returning the device close to its initial state.

nominal sample structure and corresponding hysteresis loops for four samples with increasing Gd spacer thickness $d$. With a thin Gd spacer, PMA is diminished as evidenced by a significant drop in the remanent magnetization ratio $M_{\mathrm{r}} / M_{\mathrm{s}}$ (Fig. 3b), but as $d$ is increased further, PMA again increases (Fig. 3c,d and see Supplementary Fig. 9 and related discussion for more details). We obtained similar results for other metal oxides including $\mathrm{Pt} / \mathrm{Co} / \mathrm{Zr} / \mathrm{ZrO}_{x}$ and $\mathrm{Pt} / \mathrm{Co} / \mathrm{Ta} / \mathrm{TaO}_{x}$, and find this behaviour to be rather general. The recovery of PMA for thicker metal overlayers differs from the behaviour reported in refs 8,9 for $\mathrm{Pt} / \mathrm{Co} / \mathrm{Al} / \mathrm{AlO}_{x}$ but is consistent with the results in ref. 29 for that system. An oscillatory dependence of PMA quite similar to our result in Supplementary Fig. 9 has also been reported for $\mathrm{Pt} / \mathrm{Co} / \mathrm{Al} / \mathrm{AlOx}$ in ref. 30. Although the origin of this non-monotonic anisotropy variation is not well understood, it is fortuitous because it allows us to determine sensitively the position and direction of motion of the oxidation front.

Figure $3 \mathrm{e}-\mathrm{h}$ shows that $V_{\mathrm{g}}$ applied to the sample in Fig. 3c completely reproduces the non-monotonic anisotropy transition exhibited by the as-deposited samples (Fig. 3a-d). With $V_{\mathrm{g}}>0$, PMA gradually increases (Fig. 3h) whereas with $V_{\mathrm{g}}<0$, PMA first decreases (Fig. 3f) then slowly increases with increasing dwell time (Fig. 3e; see also Supplementary Fig. 5). The effect of positive (negative) $V_{\mathrm{g}}$ is thus equivalent to increasing (decreasing) the spacing between Co and the oxidation front (see Supplementary Information for more details).

The results in Figs 2 and 3 demonstrate that these pronounced, non-volatile MAE changes occur through a thermally activated process, consistent with voltage-induced $\mathrm{O}^{2-}$ migration as observed in Fig. 1. The magnitude of the effect, which yields in Fig. $2 \mathrm{~g}-\mathrm{k}$ a change in interfacial PMA $>0.6 \mathrm{erg} \mathrm{cm}{ }^{-2}$, or $\sim 5,000 \mathrm{fJ} \mathrm{V}^{-1} \mathrm{~m}^{-1}$, corresponds to the largest magneto-electric coupling efficiency yet reported. Although the voltage response in Fig. 2 is rather slow, because the timescale for voltage-driven ion migration exhibits a super-exponential dependence on temperature and electric field ${ }^{31}$, faster magneto-ionic switching should be readily achieved by further increasing $T$ and $V_{\mathrm{g}}$. Figure $3 \mathrm{f}$ shows that indeed at $T=120^{\circ} \mathrm{C}$ and $V_{\mathrm{g}}=12 \mathrm{~V}$, the timescale for anisotropy switching drops to $<10 \mathrm{~ms}$, compared with $\sim 100 \mathrm{~s}$ at $100^{\circ} \mathrm{C}$ and $6 \mathrm{~V}$, and in contrast to the inaccessibly long timescales required at room temperature in these devices.

To achieve similar response at room temperature and low $V_{\mathrm{g}}$, we decreased the gate-oxide and -electrode thicknesses to reduce the $\mathrm{O}^{2-}$ diffusion barrier. Figure 4 summarizes results for a $\mathrm{Ta}(4 \mathrm{~nm}) / \mathrm{Pt}(3 \mathrm{~nm}) / \mathrm{Co}(0.9 \mathrm{~nm}) / \mathrm{GdO}_{x}(3 \mathrm{~nm})$ film on which thinner $\mathrm{Ta}(1.5 \mathrm{~nm}) / \mathrm{Au}(5 \mathrm{~nm})$ gate electrodes were sputter-deposited through a shadow mask (Fig. 4a). Figure 4d shows hysteresis loops measured at the electrode centre in the virgin state and after negative and positive voltage application, respectively, at room temperature. Here, PMA can be completely removed and restored by applying $V_{\mathrm{g}}= \pm 2 \mathrm{~V}$ for just $\sim 10 \mathrm{~s}$, ten times faster than for the devices in Fig. 2 at higher $T$ and $V_{\mathrm{g}}$. These results show that a substantial reduction in the effective activation barrier for ionic motion can be achieved simply by optimizing the layer thicknesses in these devices.

To quantify the voltage-induced MAE change, we determined the anisotropy field $H_{\mathrm{k}}$ by measuring the out-of-plane magnetization $M_{z}$ versus in-plane field $H_{x}$ using a MOKE lock-in technique (Supplementary Information). Figure $4 \mathrm{e}$ shows data in the virgin state and after applying $V_{\mathrm{g}}<0$ such that PMA is reduced but the easy axis remains out-of-plane. In this case, both curves correspond to the easy-axis magnetization versus hard-axis applied field, which were fitted using the Stoner-Wohlfarth model to extract $H_{\mathrm{k}}$. We find $H_{\mathrm{k}}=13 \mathrm{kOe}$ in the virgin state and $H_{\mathrm{k}}=700$ Oe after 


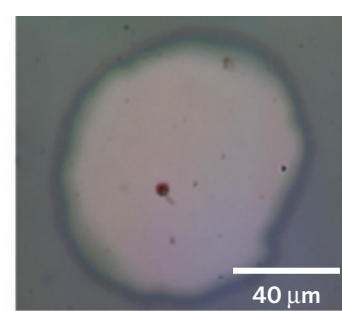

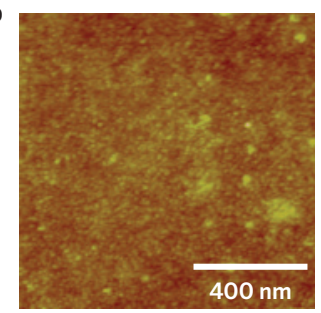

c

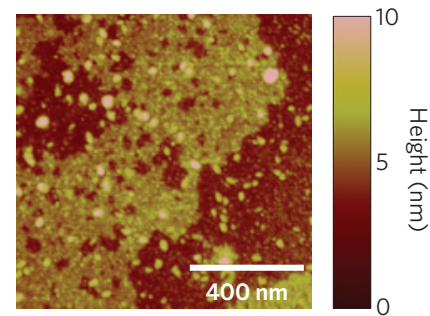

d

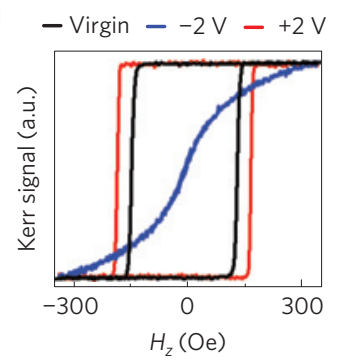

- Virgin $-2 \mathrm{~V}$

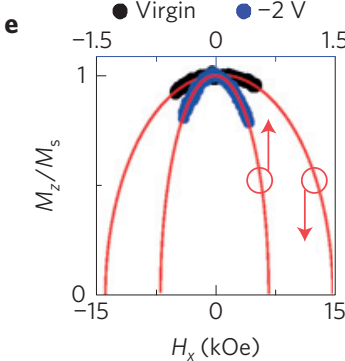

$H_{x}(\mathrm{kOe})$ f

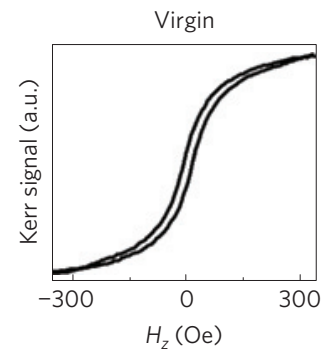

g $-+2 \mathrm{~V}--1.5 \mathrm{~V}-+2 \mathrm{~V}$

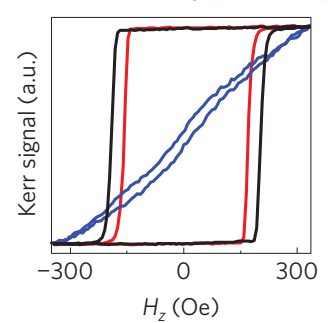

h
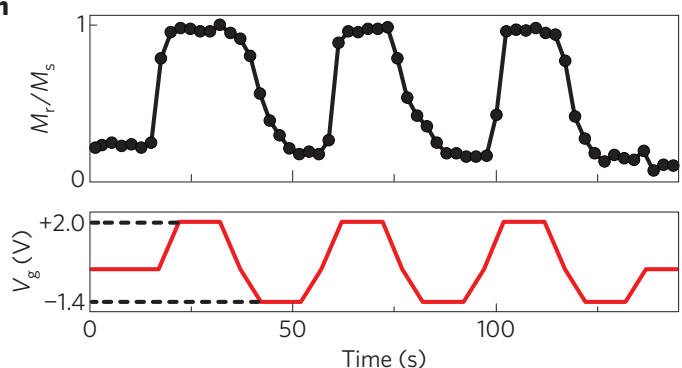

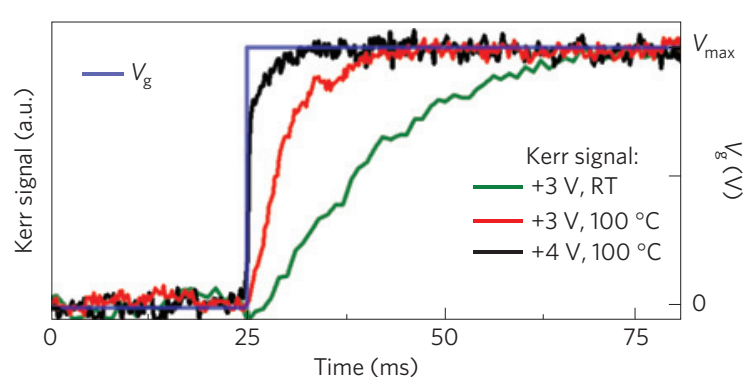

Figure 4 | Fast anisotropy switching by engineering electrode and oxide. a, Optical micrograph showing shadow-masked $100-\mu m-d i a m e t e r$

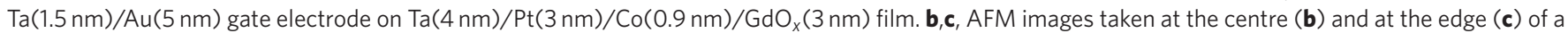

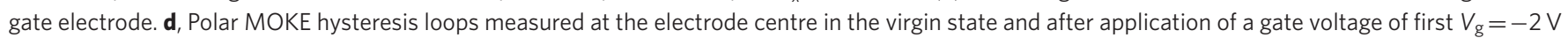

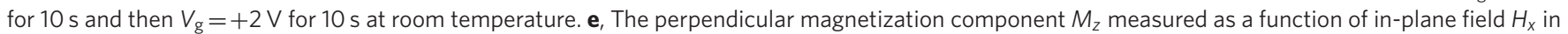
the virgin state (black points; with field scale on bottom axis) and after application of $V_{g}=-2 \mathrm{~V}$ at room temperature (blue points; with field scale on top

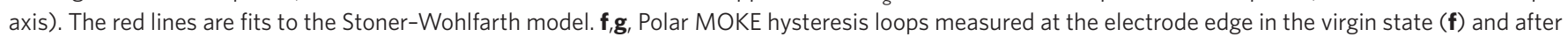
subsequent application of $V_{g}=+2 \mathrm{~V}, V_{g}=-1.5 \mathrm{~V}$ and $V_{g}=+2 \mathrm{~V}$ for 10 s each at room temperature $(\mathbf{g})$. $\mathbf{h}$, Three voltage-induced switching cycles of the

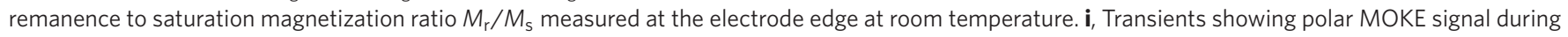
application of a $V_{g}$ pulse at room temperature (RT) and at $100{ }^{\circ} \mathrm{C}$. A perpendicular bias field of $\mathrm{H}_{z}=40$ Oe is applied to orient the magnetization in a well-defined direction as the voltage generates PMA. $V_{\max }$ refers to the amplitude of the voltage pulse.

bias application, corresponding to a MAE reduction of at least $0.75 \mathrm{erg} \mathrm{cm}^{-2}$.

Surprisingly, we found that even without voltage application, PMA is spontaneously diminished near the electrode perimeter (Fig. 4f) after exposure to ambient conditions for $\sim 24 \mathrm{~h}$, suggesting that the oxygen affinity of the underlying Co layer is alone sufficient to drive $\mathrm{O}^{2-}$ through the $\mathrm{GdO}_{x}$ there (see Supplementary Information for more details). This behaviour was restricted, however, to a $\sim 10 \mu \mathrm{m}$ region at the electrode perimeter, whereas at the electrode interior and far from the electrodes, PMA is stable with time. Atomic force microscopy (AFM) shows that near its centre the electrode is continuous (Fig. 4b), but shadowing during deposition leads to a thinner region at the edge where the $\mathrm{Au}$ is electrically contiguous but structurally porous (Fig. 4c) owing to percolated island growth. This porous microstructure should extend the lateral extent of the triple-phase boundary, where gas phase, electrode and electrolyte coincide. Although these room-temperature effects are probably mainly aided by the reduced Au thickness and extended triple-phase boundary in these devices, the impact of reduced $\mathrm{GdO}_{x}$ film thickness on $\mathrm{O}^{2-}$ mobility cannot, at this time, be excluded.

Figure $4 \mathrm{~g}$ shows that the spontaneous loss in PMA in this region (Fig. 4f) can be reversed under positive $V_{\mathrm{g}}$ (Fig. 4g), consistent with $\mathrm{O}^{2-}$ migration away from the Co, and that PMA can be toggled repeatedly (Fig. 4g,h). As Co redox reactions are markedly enhanced near the triple-phase boundary in these devices, it follows that faster voltage-induced switching might likewise be attained. Figure 4i shows time-resolved measurements of voltageinduced MAE switching at the electrode perimeter that directly yield the switching speed. Here, starting from the low-anisotropy state (similar to Fig. 4f), a voltage step $V_{\mathrm{g}}>0$ was applied to generate PMA while the time-resolved polar MOKE signal was measured. A small perpendicular bias field was simultaneously applied, so that as the MAE transitions from in-plane to out-of-plane, the magnetization orients in a well-defined direction. This field is too weak by itself to orient the magnetization out of the plane in the initial state, and is used only to break the symmetry so that when the easy axis switches to out-of-plane, the magnetization prefers the 'up' state over the 'down' state.

Figure $4 \mathrm{i}$ shows that on voltage application, the polar MOKE signal rapidly rises and saturates, indicating tilting of the magnetization out of the plane as the film transitions to PMA. The MOKE transient for $V_{\mathrm{g}}=+3 \mathrm{~V}$ at room temperature has a rise time of $\sim 25 \mathrm{~ms}$, similar to the switching time achieved in Fig. $3 \mathrm{f}$ at $\mathrm{T}=120^{\circ} \mathrm{C}$ and $V_{\mathrm{g}}=+12 \mathrm{~V}$, where the $\mathrm{GdO}_{x}$ and Au electrodes 

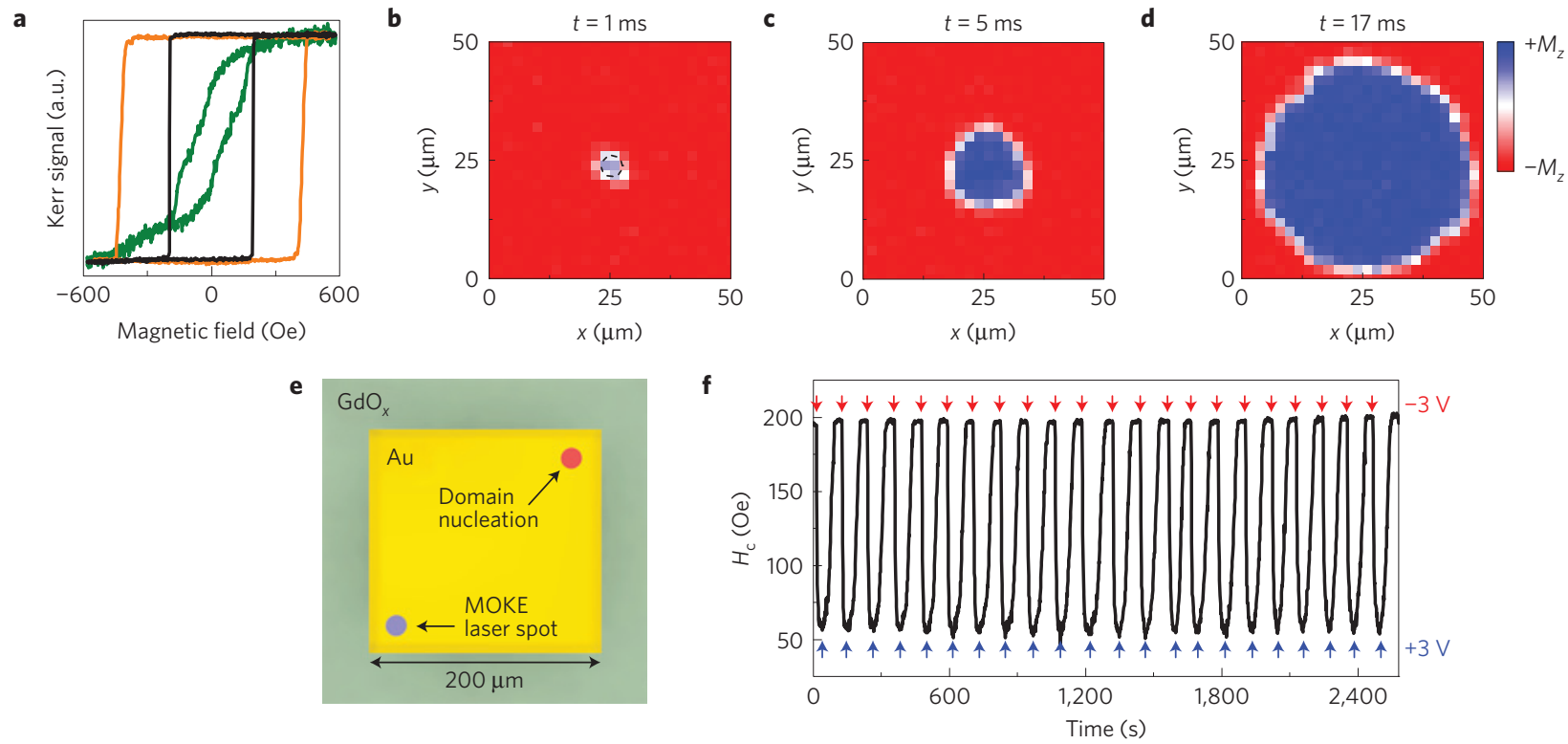

Figure 5 | Effects of voltage and laser illumination on magnetic anisotropy. a, Polar MOKE hysteresis loops measured inside a gate electrode with the device in its virgin state (black line), after application of a gate voltage $V_{g}=-3 \mathrm{~V}$ for $90 \mathrm{~s}$ (orange line) and after application of $V_{g}=-7 \mathrm{~V}$ for $180 \mathrm{~s}$ under

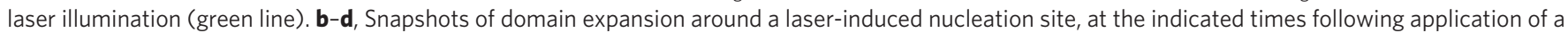
reverse field step of $90 \mathrm{Oe}$ at $t=0$. All snapshots were acquired at zero bias. The dashed black line in $\mathbf{b}$ outlines the area exposed for $100 \mathrm{~s}$ to the $10 \mathrm{~mW}$ laser spot at a gate voltage $-3 \mathrm{~V}$. e, Schematic showing top view of an electrode in which a laser-induced nucleation site has been created at the

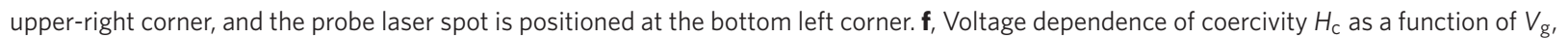
corresponding to the schematic experiment geometry in $\mathbf{e}$, as $V_{g}$ is cycled between $\pm 3 \mathrm{~V}$.

were much thicker. The switching time is further reduced by increasing $T$ and $V_{g}$, dropping to $\sim 700 \mu$ s at $100^{\circ} \mathrm{C}$ and $+4 \mathrm{~V}$. The remarkable increase in switching speed, by $\sim 6$ orders of magnitude compared with the same $T$ and $V_{\mathrm{g}}$ in Fig. 2, together with the unprecedented degree by which the MAE can be switched, indicates that magneto-ionic coupling offers a promising new route towards magneto-electric devices.

On the basis of these findings we demonstrate local MAE patterning using the MOKE laser spot to locally heat the sample and activate oxygen migration under $V_{\mathrm{g}}$. Using the devices in Fig. 2b, we first apply $V_{\mathrm{g}}<0$ at room temperature to create a potential well at the electrode perimeter, evidenced by a jump in $H_{c}$ beneath the electrode (Fig. 5a). This isolates the electrode area from DW motion in the adjacent Co film. With $V_{\mathrm{g}}$ applied, we then increase the MOKE laser power $P$ from $1 \mathrm{~mW}$ to $10 \mathrm{~mW}$, corresponding to a local temperature rise of $\sim 20^{\circ} \mathrm{C}$ (Methods). This causes $H_{\mathrm{c}}$ and $M_{\mathrm{r}} / M_{\mathrm{s}}$ to drop markedly underneath the spot (Fig. 5a), and this change is retained when $V_{\mathrm{g}}$ is removed and $P$ decreased to $1 \mathrm{~mW}$. Neither $V_{\mathrm{g}}$ nor high $P$ alone is sufficient to cause irreversible changes, but when both are sufficiently high (Supplementary Fig. 12), laser-induced heating activates voltage-driven $\mathrm{O}^{2-}$ migration, facilitating local MAE imprinting (we note that the $1 \mathrm{~mW}$ incident power used in the devices with thinner $\mathrm{Ta} / \mathrm{Au}$ electrodes in Fig. 4 was below the threshold for laser-assisted effects for those structures).

Owing to the local anisotropy reduction, the illuminated spot acts as a domain nucleation site, as seen in the time-resolved scanning MOKE images in Fig. 5b-d (Methods). To demonstrate the reversibility of this MAE imprinting, we first created a laserinduced DW nucleation site in one corner of an electrode and then placed the laser spot in the diagonally opposite corner (Fig. 5e) with $P=10 \mathrm{~mW}$. At $V_{\mathrm{g}}=0$ the higher laser power alone has no effect on $H_{c}$, which is determined by the field necessary to propagate a DW from the far corner. However, with $V_{\mathrm{g}}=-3 \mathrm{~V}, H_{\mathrm{c}}$ and $M_{\mathrm{r}} / M_{\mathrm{s}}$ drop markedly, indicating that instead of propagating across the electrode, DWs nucleate directly underneath the laser spot owing to the local PMA reduction. Positive bias restores $M_{\mathrm{r}} / M_{\mathrm{s}}$ and $H_{\mathrm{c}}$ to their initial values and $H_{c}$ can be toggled repeatedly in this manner as $V_{\mathrm{g}}$ is cycled between $+3 \mathrm{~V}$ and $-3 \mathrm{~V}$ (Fig. $5 \mathrm{f}$ ).

Finally, we imprint more complex anisotropy patterns that allow for spatial control of magnetization dynamics. In Fig. 6, we define a conduit in which DWs are injected from a laser-written nucleation site and propagate along a pre-defined path. At $V_{\mathrm{g}}=-3 \mathrm{~V}$, a point inside the electrode area was illuminated at $P=10 \mathrm{~mW}$ for $100 \mathrm{~s}$ to produce a DW nucleation site. The laser was then scanned along an L-shaped line in $1.25 \mu \mathrm{m}$ steps with a variable dwell time to produce a conduit of reduced MAE (Fig. 6a). Figure 6a-f shows time-resolved MOKE images of field-driven domain expansion in the patterned region (Methods). Here, a reverse domain nucleates at the laser-defined nucleation site (Fig. 6b) and expands preferentially along the laser-written conduit (Fig. $6 \mathrm{c}-\mathrm{f}$ ). The degree of confinement depends on the difference in MAE in the film and in the patterned region, which determines the difference in creep velocity along and orthogonal to the conduit. The DW velocity follows $v \propto \exp \left(-E_{\mathrm{a}}(H) / k_{\mathrm{B}} T\right)$, where the activation energy $E_{\mathrm{a}}(H) \propto H^{-1 / 4}$ depends on the MAE (ref. 27). Figure $6 \mathrm{~g}$ shows that the slope of $\ln (v)$ versus $H^{-1 / 4}$, and hence the activation energy that determines the DW velocity, can be precisely tuned to control the DW dynamics. By reducing the anisotropy in the conduit we enhance the velocity by up to a factor of $\sim 160$. The MAE can be further reduced, but in this case nucleation along the conduit is observed.

We note that local MAE patterning has previously been demonstrated using local ion beam irradiation ${ }^{32,33}$, but has never been realized in a non-destructive and completely reversible way. Here, the spatial resolution is limited by the laser spot size to a few micrometres. However, this resolution limit could easily be overcome by instead heating the sample globally and writing the anisotropy pattern locally using, for example, a conductive AFM tip to apply $V_{\mathrm{g}}$ with high spatial resolution.

Our work shows that interfacial chemistry in metal/metaloxide bilayers can be electrically gated using an all-solid-state 


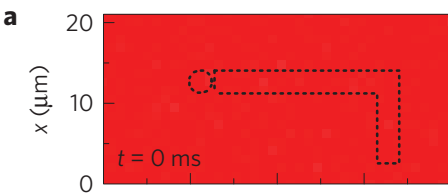

b
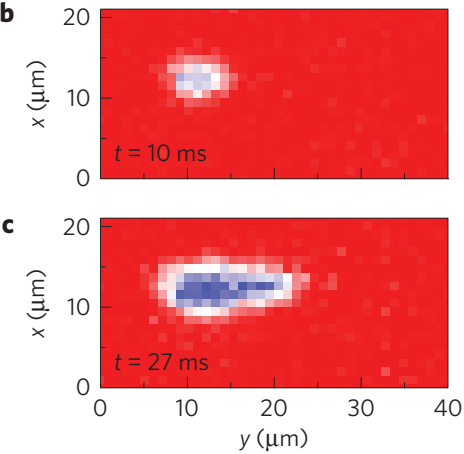

d
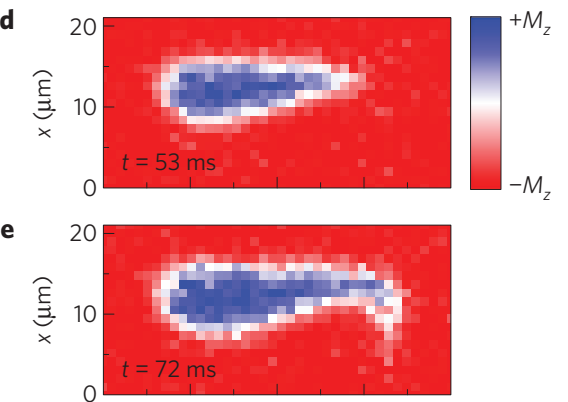

f

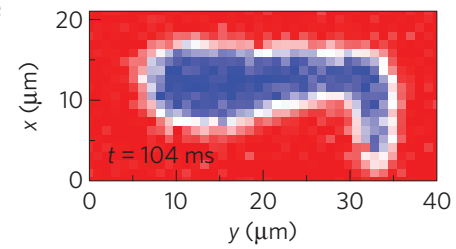

g

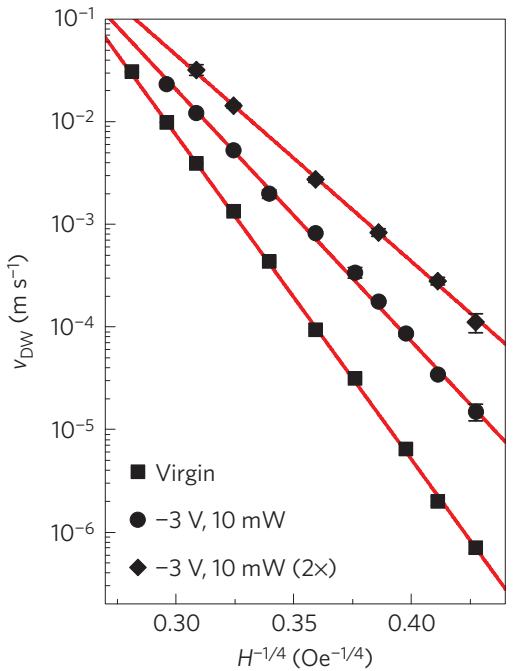

Figure 6 | Laser-defined anisotropy patterns and DW conduits. a-f, Time-resolved polar MOKE maps showing domain expansion in a laser-defined DW conduit with increasing time $t$ after application of a magnetic driving field $H=42$ Oe. Dashed black lines in a outline the area illuminated by the laser spot with $10 \mathrm{~mW}$ incident power, under gate voltage $V_{g}=-3 \mathrm{~V}$. The dashed circle was exposed for $100 \mathrm{~s}$ whereas the dashed, $\mathrm{L}$-shaped line was scanned in $1.25 \mu \mathrm{m}$ steps with $65 \mathrm{~s}$ exposure at each point. All maps were acquired under zero bias. $\mathbf{g}$, DW velocity as a function of magnetic field $H$ in virgin film and along a conduit exposed at $10 \mathrm{~mW}$ incident power under $V_{\mathrm{g}}=-3 \mathrm{~V}$ for $60 \mathrm{~s}$ and $65 \mathrm{~s}$.

device, operating at low voltage and within the typical operating temperature range of common semiconductor electronics. Specifically for $\mathrm{Co} /$ metal-oxide bilayers, where interfacial MAE is sensitive to interface oxygen coordination, we use voltage control of oxygen stoichiometry to achieve unprecedented control over magnetic anisotropy. Moreover, we show that relatively small changes in temperature and gate voltage can improve device response times by orders of magnitude, and that by simply varying the thickness and morphology of the gate oxide and electrode, the magneto-ionic switching time drops from hundreds of seconds to hundreds of microseconds. Therefore, considerable further improvements in performance and functionality can probably be anticipated by examining oxides with higher ionic conductivity such as yttria-stabilized zirconia ${ }^{15}$, or designing gate-oxide heterostructures that include separately optimized oxygen storage and ion conducting layers. Although this work focused on magnetic properties, reversible voltage-gated control of oxygen stoichiometry in metal/metal-oxide bilayers makes a wide range of materials properties and effects amenable to solid-state electrical control. These results thus suggest a path towards electrically gating a variety of phenomena governed by metal/oxide interfaces, and provide a new means to locally and reversibly imprint material properties by local activation of ionic migration.

\section{Methods}

Sample preparation. $\mathrm{Ta}(4 \mathrm{~nm}) / \mathrm{Pt}(3 \mathrm{~nm}) / \mathrm{Co}(0.9 \mathrm{~nm}) / \mathrm{GdO}_{x}(3 \mathrm{~nm})$ films were prepared by d.c. magnetron sputtering at room temperature under 3 mtorr $\mathrm{Ar}$ with a background pressure of $\sim 1 \times 10^{-7}$ torr, on thermally oxidized $\mathrm{Si}(100)$ substrates. For the samples described in Fig. 3, the top $\mathrm{GdO}_{x}$ layer was $30 \mathrm{~nm}$ thick. All $\mathrm{GdO}_{x}$ layers were deposited by reactive sputtering from a metal Gd target at an oxygen partial pressure of $\sim 5 \times 10^{-5}$ torr. Gate electrodes of $\mathrm{GdO}_{x}(30 \mathrm{~nm}) / \mathrm{Ta}(2 \mathrm{~nm}) / \mathrm{Au}(12 \mathrm{~nm})$ were patterned using electron-beam lithography and lift-off. For the samples with the 30 -nm-thick $\mathrm{GdO}_{x}$ top layer described in Fig. 3, the $\mathrm{Ta}(2 \mathrm{~nm}) / \mathrm{Au}(12 \mathrm{~nm})$ electrodes were deposited through a shadow mask.

In situ high-resolution TEM characterization. Microstructural analysis and EELS were performed on a JEOL 2200FS TEM with double Cs correctors, operated at $200 \mathrm{keV}$. A cross-sectional TEM specimen was fabricated from a patterned $\mathrm{Si} / \mathrm{SiO}_{2} / \mathrm{Ta}(4 \mathrm{~nm}) / \mathrm{Pt}(3 \mathrm{~nm}) / \mathrm{Co}(0.9 \mathrm{~nm}) / \mathrm{GdO}_{x}(30 \mathrm{~nm}) / \mathrm{Ta} / \mathrm{Au}$ sample using the following steps. First, a Si substrate was glued to the top surface of the sample and the $\mathrm{Si} /$ multilayer/Si sandwich was subsequently cut into thin slices.
Next, a thin slice was polished into a wedge by a MultiPrep polishing machine (Allied High-Tech). After gluing the specimen to a half TEM Cu grid, it was further polished by Ar ion milling. Before mounting the grid onto an in situ electrical probing holder (HE150, Nanofactory Instruments AB), the Si was unglued from the wedge using acetone. After Si removal, a piezo-controlled $\mathrm{Pt} / \mathrm{Ir}$ tip with a diameter of about $40 \mathrm{~nm}$ was able to contact the patterned electrode on top of the $\mathrm{GdO}_{x}$ layer. Slight bending of the sample was observed after contact, but the structural integrity of the layers remained intact (Fig. 1a, inset). Silver paste was used to make electrical contact between the bottom electrode of the layer structure and the $\mathrm{Cu}$ grid (Supplementary Fig. 1). The thickness of the TEM specimen was estimated to be less than $30 \mathrm{~nm}$ by measuring the intensity ratio of the plasmon loss and the zero-loss peaks in EELS. For the analysis of EELS core-loss peaks, background subtraction was performed using a power-law fit. The lateral resolution of STEM-EELS characterization was about $0.25 \mathrm{~nm}$.

MOKE measurements. Polar MOKE measurements were made using a $532 \mathrm{~nm}$ diode laser attenuated to $1 \mathrm{~mW}$, except where noted. The laser was focused to a $\sim 3-\mu \mathrm{m}$-diameter probe spot and positioned by a high-resolution $(50 \mathrm{~nm})$ scanning stage with integrated temperature control. Gate voltage was applied using a mechanically compliant $\mathrm{BeCu}$ microprobe. Mechanically generated nucleation sites created for the measurements in Fig. 2 were prepared by applying mechanical stress to the film surface using a stiff W microprobe tip. Magnetic hysteresis loops were measured at a sweep rate of $28.3 \mathrm{kOe} \mathrm{s}^{-1}$, using an electromagnet with a rise time of $\sim 300 \mu$ s and a maximum amplitude of 650 Oe.

The time-resolved domain expansion snapshots in Figs 5 and 6 were obtained by, at each pixel, first saturating the magnetization and then applying a reverse field step ( $H=90 \mathrm{Oe}$ in Fig. 5 , and $H=42 \mathrm{Oe}$ in Fig. 6) while acquiring a time-resolved MOKE signal transient. Five reversal cycles were averaged at each pixel, from which the average trajectory of the expanding domain was reconstructed.

Time-resolved MOKE transients along a line extending radially from a nucleation site were used to determine the DW velocity reported in Fig. $6 \mathrm{~g}$. At each position, 25 reversal cycles were acquired and averaged, yielding the cumulative probability distribution of switching times. The mean reversal time $t_{1 / 2}$, taken as the time at which the probability of magnetization switching is $50 \%$, was plotted versus position, and the slope used to determine the mean velocity.

Laser-induced temperature rise. To estimate the laser-induced temperature increase $\Delta T$, we used the temperature dependence of the coercivity of a submicrometre patterned feature. We first measured $H_{\mathrm{c}}$ versus substrate temperature, using a temperature-controlled stage and a low incident laser power $(<1 \mathrm{~mW})$ for the MOKE probe spot, and then measured $H_{\mathrm{c}}$ versus incident laser power $P$, at a fixed substrate temperature. We estimate that $P=1 \mathrm{~mW}$ corresponds to a negligible $\Delta T$ whereas $P=10 \mathrm{~mW}$ corresponds to a $\Delta T$ of at least $\sim 20^{\circ} \mathrm{C}$. 
Received 24 March 2014; accepted 8 October 2014; published online 17 November 2014

\section{References}

1. Stair, P. C. Metal-oxide interfaces where the action is. Nature Chem. 3, 345-346 (2011)

2. Yamada, Y. et al. Nanocrystal bilayer for tandem catalysis. Nature Chem. 3 , 372-376 (2011)

3. Yang, J. J., Strukov, D. B. \& Stewart, D. R. Memristive devices for computing. Nature Nanotech. 8, 13-24 (2013).

4. Waser, R., Dittmann, R., Staikov, G. \& Szot, K. Redox-based resistive switching memories-nanoionic mechanisms, prospects, and challenges. Adv. Mater. 21, 2632-2663 (2009).

5. Jeong, J. et al. Suppression of metal-insulator transition in $\mathrm{VO}_{2}$ by electric field-induced oxygen vacancy formation. Science 339, 1402-1405 (2013).

6. Ikeda, S. et al. A perpendicular-anisotropy $\mathrm{CoFeB}-\mathrm{MgO}$ magnetic tunnel junction. Nature Mater. 9, 721-724 (2010)

7. Miron, I. M. et al. Current-driven spin torque induced by the Rashba effect in a ferromagnetic metal layer. Nature Mater. 9, 230-234 (2010).

8. Manchon, A. et al. X-ray analysis of the magnetic influence of oxygen in $\mathrm{Pt} / \mathrm{Co} / \mathrm{AlO}_{x}$ trilayers. J. Appl. Phys. 103, 07A912 (2008).

9. Rodmacq, B., Manchon, A., Ducruet, C., Auffret, S. \& Dieny, B. Influence of thermal annealing on the perpendicular magnetic anisotropy of $\mathrm{Pt} / \mathrm{Co} / \mathrm{AlO}_{x}$ trilayers. Phys. Rev. B 79, 024423 (2009).

10. Zhang, W. \& Smith, J. R. Nonstoichiometric interfaces and $\mathrm{Al}_{2} \mathrm{O}_{3}$ adhesion with $\mathrm{Al}$ and Ag. Phys. Rev. Lett. 85, 3225-3228 (2000).

11. Howe, J. M. Bonding, structure, and properties of metal-ceramic interfaces .1. Chemical bonding, chemical-reaction, and interfacial structure. Int. Mater. Rev. 38, 233-256 (1993)

12. Padture, N. P., Gell, M. \& Jordan, E. H. Thermal barrier coatings for gas-turbine engine applications. Science 296, 280-284 (2002).

13. Losego, M. D., Grady, M. E., Sottos, N. R., Cahill, D. G. \& Braun, P. V. Effects of chemical bonding on heat transport across interfaces. Nature Mater. 11 502-506 (2012)

14. Maier, J. Nanoionics: Ion transport and electrochemical storage in confined systems. Nature Mater. 4, 805-815 (2005).

15. Adler, S. B. Factors governing oxygen reduction in solid oxide fuel cell cathodes. Chem. Rev. 104, 4791-4843 (2004).

16. Maruyama, T. et al. Large voltage-induced magnetic anisotropy change in a few atomic layers of iron. Nature Nanotech. 4, 158-161 (2009)

17. Wang, W. G., Li, M. G., Hageman, S. \& Chien, C. L. Electric-field-assisted switching in magnetic tunnel junctions. Nature Mater. 11, 64-68 (2012).

18. Shiota, Y. et al. Induction of coherent magnetization switching in a few atomic layers of FeCo using voltage pulses. Nature Mater. 11, 39-43 (2012).

19. Duan, C. G. et al. Surface magnetoelectric effect in ferromagnetic metal films. Phys. Rev. Lett. 101, 137201 (2008).

20. Bauer, U., Przybylski, M., Kirschner, J. \& Beach, G. S. D. Magnetoelectric charge trap memory. Nano Lett. 12, 1437-1442 (2012).

21. Rajanikanth, A., Hauet, T., Montaigne, F., Mangin, S. \& Andrieu, S. Magnetic anisotropy modified by electric field in $\mathrm{V} / \mathrm{Fe} / \mathrm{MgO}(001) / \mathrm{Fe}$ epitaxial magnetic tunnel junction. Appl. Phys. Lett. 103, 062402 (2013).

22. Bauer, U., Emori, S. \& Beach, G. S. D. Electric field control of domain wall propagation in $\mathrm{Pt} / \mathrm{Co} / \mathrm{GdO}_{x}$ films. Appl. Phys. Lett. 100, 192408 (2012)
23. Bauer, U., Emori, S. \& Beach, G. S. D. Voltage-controlled domain wall traps in ferromagnetic nanowires. Nature Nanotech. 8, 411-416 (2013).

24. Bonell, F. et al. Reversible change in the oxidation state and magnetic circular dichroism of Fe driven by an electric field at the $\mathrm{FeCo} / \mathrm{MgO}$ interface. Appl. Phys. Lett. 102, 152401 (2013).

25. Tournerie, N., Engelhardt, A. P., Maroun, F. \& Allongue, P. Influence of the surface chemistry on the electric-field control of the magnetization of ultrathin films. Phys. Rev. B 86, 104434 (2012).

26. Reichel, L., Oswald, S., Fahler, S., Schultz, L. \& Leistner, K. Electrochemically driven variation of magnetic properties in ultrathin CoPt films. J. Appl. Phys. 113, 143904 (2013).

27. Bauer, U., Emori, S. \& Beach, G. S. D. Voltage-gated modulation of domain wall creep dynamics in an ultrathin metallic ferromagnet. Appl. Phys. Lett. 101, 172403 (2012)

28. Balluffi, R. W., Allen, S. M. \& Carter, W. C. Kinetics of Materials 209-228 (John Wiley, 2005).

29. Lacour, D. et al. Magnetic properties of postoxidized $\mathrm{Pt} / \mathrm{Co} / \mathrm{Al}$ layers with perpendicular anisotropy. Appl. Phys. Lett. 90, 192506 (2007).

30. Dahmane, Y. et al. Oscillatory behavior of perpendicular magnetic anisotropy in $\mathrm{Pt} / \mathrm{Co} / \mathrm{Al}(\mathrm{O}-\mathrm{x})$ films as a function of $\mathrm{Al}$ thickness. Appl. Phys. Lett. 95, $222514(2009)$

31. Strukov, D. B. \& Williams, R. S. Exponential ionic drift: Fast switching and low volatility of thin-film memristors. Appl. Phys. A 94, 515-519 (2009).

32. Chappert, C. et al. Planar patterned magnetic media obtained by ion irradiation. Science 280, 1919-1922 (1998).

33. Franken, J. H., Swagten, H. J. M. \& Koopmans, B. Shift registers based on magnetic domain wall ratchets with perpendicular anisotropy. Nature Nanotech. 7, 499-503 (2012)

\section{Acknowledgements}

This work was supported by the National Science Foundation under NSF-ECCS -1128439 and through the MRSEC Program under DMR-0819762, and by the Samsung Global MRAM Innovation program. Technical support from D. Bono, M. Tarkanian and E. Shaw is gratefully acknowledged. Work was performed using instruments in the MIT Nanostructures Laboratory, the Scanning Electron-Beam Lithography facility at the Research Laboratory of Electronics, and the Center for Materials Science and Engineering at MIT. In situ TEM and EELS characterization was conducted using the facilities of the Aalto University Nanomicroscopy Center (Aalto-NMC) in Finland.

\section{Author contributions}

U.B. and G.S.D.B. conceived and designed the experiments. H.L.T. proposed the extension of studies to higher temperatures. U.B. prepared the samples with help from A.J.T. and S.E. U.B. performed the MOKE experiments and analysed the data. P.A. and U.B. conducted the VSM and AFM measurements. S.v.D. and L.Y. performed and analysed the TEM and EELS measurements. U.B. wrote the manuscript with assistance from G.S.D.B. and input from S.v.D. and L.Y. All authors discussed the results.

\section{Additional information}

Supplementary information is available in the online version of the paper. Reprints and permissions information is available online at www.nature.com/reprints. Correspondence and requests for materials should be addressed to G.S.D.B.

\section{Competing financial interests}

The authors declare no competing financial interests. 